\title{
SOME REMARKS ON SELF-DUAL LOCALLY COMPACT ABELIAN GROUPS
}

\author{
BY \\ LAWRENCE CORWIN( $\left.{ }^{1}\right)$
}

\begin{abstract}
The main results of this paper are the construction of some new selfdual locally compact Abelian groups and the proof of a structure theorem for a certain class of such groups. The construction is based on an investigation of when the extension of a compact Abelian group by its dual yields a self-dual group. It turns out that such extensions can be described algebraically; the structure theorem follows from an analysis of the algebraic description.
\end{abstract}

1. Introduction. Not much is known about the structure of self-dual locally compact Abelian groups; in particular, there are not many known ways to construct them. The following list (modified slightly from [3, p. 422]) seems to include all standard examples:

1. $\boldsymbol{R}^{n}$ (for finite $n$ ) is self-dual; moreover, if $G$ is any LCA group and we write $G \cong \boldsymbol{R}^{n} \oplus G_{0}$, where $G_{0}$ contains a compact open subgroup, then $G$ is self-dual iff $G_{0}$ is. (A proof of this last statement will be given later.)

Henceforth, therefore, we shall assume that $G$ contains a compact open subgroup.

2. $\boldsymbol{Q}_{p}$ (for all primes $p$ ) and the finite cyclic groups with $n$ elements, $C(n)$, are self-dual.

3. If $G$ is any LCA group and $G^{*}$ is its dual, then $G \oplus G^{*}$ is self-dual.

4. The direct sum of finitely many self-dual groups is self-dual.

5. Let $G_{\alpha}(\alpha \in \mathscr{A})$ be a self-dual group, let $f_{\alpha}: G_{\alpha} \rightarrow G_{\alpha}^{*}$ be the isomorphism, and let $H_{\alpha} \subseteq G_{\alpha}$ be a compact open subgroup such that $H_{\alpha}^{\perp}=f_{\alpha}\left(H_{\alpha}\right)$. Then the restricted direct product $\prod_{\alpha \in \mathscr{A}}^{\prime}\left(G_{\alpha} ; H_{\alpha}\right)$ of the $G_{\alpha}$ with respect to the $H_{\alpha}$ is selfdual. The known examples for $\left(G_{\alpha} ; H_{\alpha}\right)$ are, essentially, $\left(Q_{p} ; Z_{p}\right),\left(C\left(p^{2 n}\right) ; C\left(p^{n}\right)\right)$, and $\left(K \oplus K^{*} ; C \oplus C^{\perp}\right)$, where $C$ is compact open in $K$.

There are two important observations to make about these groups:

(A) In each case, the isomorphism $f$ of $G$ with $G^{*}$ can be made self-adjoint. (The adjoint $f^{*}$ of $f$ is the map from $G$ to $G^{*}$ defined by $\left\langle f^{*}\left(g_{1}\right), g_{2}\right\rangle=\left\langle g_{1}, f\left(g_{2}\right)\right\rangle$ for all $g_{1}, g_{2} \in G$.) In cases 4 and 5 , this statement is true if all the $f_{\alpha}$ are

Received by the editors March 18, 1969 and, in revised form, September 16, 1969.

AMS Subject Classifications. Primary 4251.

Key Words and Phrases. Locally compact Abelian group, self-dual, group extension, cohomology of groups.

(1) Research partially sponsored by the Air Force Office of Scientific Research, Office of Aerospace Research, under contract no. F44620-67-Z-0029.

Copyright (C) 1970, American Mathematical Society 
self-adjoint; that can be arranged in every known case. In what follows, this property of $f$ will be assumed.

(B) In each case, $G$ can be written as $G_{1} \times G_{2}$, where $G_{2}$ is finite and $G_{1}$ is a selfdual group with a compact subgroup isomorphic to its own annihilator. (Recall that $G$ has a compact open subgroup.) In what follows, we shall assume for convenience that $G$ itself contains a compact subgroup isomorphic to its own annihilator.

Some remarks about hypotheses (A) and (B) may be in order. It is not hard to devise examples of self-dual groups with non-self-adjoint isomorphisms; I do not know, however, of an example for which there is not also a self-adjoint isomorphism. As for (B), it seems a quite reasonable conjecture that it is a consequence of (A).

Even with hypotheses (A) and (B), it is not true that the constructions 1-5 suffice to find all self-dual groups, as we shall see. However, one can find a general construction, based on the following result:

THEOREM 1.1. Let $f: G_{0} \rightarrow G_{0}^{*}$ be a self-adjoint isomorphism and let $H \subseteq G_{0}$ be a closed subgroup with $H^{\perp} \subseteq f(H)$. Then $H / f^{-1}\left(H^{\perp}\right)$ is self-dual under the self-adjoint map $f^{\sim}$ induced by $f$.

Proof. $\mathrm{G} / f^{-1}\left(H^{\perp}\right)$ has as dual $\left[f^{-1}\left(H^{\perp}\right)\right]^{\perp}=f(H)$; hence $H / f^{-1}\left(H^{\perp}\right)$ has as dual $f(H) /\left(H / f^{-1}\left(H^{\perp}\right)\right)^{\perp}=f(H) / H^{\perp}$. The self-adjointness of $F$ is easy to check.

We shall show that for any self-dual group $G$ satisfying hypotheses (A) and (B), there exists a group $G_{0}$, of a reasonably well-specified type, and a subgroup $H$ of $G_{0}$ such that $G=f(H) / f^{-1}\left(H^{1}\right)$, as in Theorem 1.1. The approach is as follows: $G$ is an extension of a compact group by its dual; we analyze the cocycle giving rise to that extension. In the process, we obtain an algebraic characterization of $G$.

All groups to be used in this paper are Abelian; with the exception of the circle group $\boldsymbol{T}$, the group operation on them is + . On some occasions, we shall regard the cyclic group with $p^{n}$ elements, $C\left(p^{n}\right)$, and the group $Z\left(p^{\infty}\right)=Z[1 / p] / Z$ as subgroups of $\boldsymbol{Q} / \boldsymbol{Z}$; the coset representatives will be understood to be in the interval $\left(-\frac{1}{2}, \frac{1}{2}\right]$. The action of $G$ on its dual is written as $\langle$,$\rangle .$

We conclude this section by showing that it was indeed legitimate to consider only the case where $G$ contains a compact open subgroup. The following theorem proves even more.

THEOREM 1.2. Suppose $G \cong \boldsymbol{R}^{n} \times G_{0} \cong \boldsymbol{R}^{m} \times G_{1}$, where $G_{0}$ and $G_{1}$ contain compact open subgroups. Then $m=n$ and $G_{0}=G_{1}$.

Proof. For a proof that $m=n$, see [3, Theorem 24.30]. The rest of the theorem is trivial when $G_{0}$ is compact; then $G_{0}=$ set of compact elements of $G$, and so $G_{0}=G_{1}$. It is also easy when $G_{0}$ is discrete; then $G_{0}$ and $G_{1}$ have one element each in every component of $G$. The general result now follows from these remarks and the 5-lemma [6, p. 85].

2. Some lemmas. The lemmas in this section will be used later in the paper; they have only limited interest in their own right. 
LEMMA 2.1. Let $G_{0}$ be an Abelian group whose torsion subgroup is a direct summand, and let $F$ be a finite Abelian group.

(a) If $G$ is such that $0 \rightarrow F \rightarrow G_{0} \rightarrow G \rightarrow 0$ is exact, then the torsion subgroup of $G$ is a direct summand.

(b) If $G$ is such that $0 \rightarrow F \rightarrow G \rightarrow G_{0} \rightarrow 0$ is exact, then the torsion subgroup of $G$ is a direct summand.

Proof. (a) is trivial, since $F \subseteq$ torsion group of $G_{0}$.

(b) The dual of the theorem is that if $G_{0}^{*}$ is the direct sum of a divisible subgroup $D$ and a totally disconnected subgroup $T$, then so is any finite extension of $G_{0}^{*}$. It suffices to prove this for an extension by a cyclic subgroup $C(n)$. The extension $G^{*}$ is defined by giving a coset representative $y$ for a generator $x$ of $C(n)$ (see $[2, \S 15.3]$, for details). Because $D$ is divisible, we may pick $y$ so that $n y \in T$. This shows that $G^{*}=D \oplus$ a cyclic extension of $T$; the extension is also totally disconnected, since $T$ is open in it. This proves the lemma.

Lemma 2.2. Suppose $G=G_{0} \oplus F=\prod_{\alpha}^{\prime}\left(G_{\alpha} ; H_{\alpha}\right)$, where $F$ is a cyclic group of order $p^{n}$. Then one of the $G_{\alpha}$ contains a cyclic direct summand of order $p^{n}$.

Proof. Let $x$ generate $F$. In terms of the restricted direct product, $x=\left(\ldots, x_{\alpha}, \ldots\right)$, say, where almost all the $x_{\alpha}$ are in $H_{\alpha}$. Now write $x_{\alpha}=y_{\alpha}+z_{\alpha}$, where $y_{\alpha} \in G_{0}$ and $z_{\alpha} \in F$. $\sum_{\alpha} x_{\alpha}=x$, and projection on $F$ is continuous; therefore $\sum_{\alpha} z_{\alpha}=x$. Since $F$ is finite, almost every $z_{\alpha}$ must be 0 and one of the nonzero terms must generate $F$. Say it is $z_{\beta}$. Then in $G_{\beta}, Z z_{\beta}$ is a cyclic subgroup of order $p^{n}$ and a direct summand (the other summand could be $\left\{a \in G_{\beta}\right.$ : the projection of $a$ on $F$ is 0$\}$ ).

Note that the same result clearly holds for a finite direct product as well.

3. Duals of Abelian extensions. Let $0 \rightarrow N \rightarrow G \rightarrow H \rightarrow 0$ be an exact sequence of Abelian topological groups, with $N$ compact and open in $G$. Then there is determined a dual exact sequence $0 \rightarrow H^{*} \rightarrow G^{*} \rightarrow N^{*} \rightarrow 0$. We begin by showing how to find (in terms of cocycles) either sequence from the other.

Let $f: H \times H \rightarrow N$ be a symmetric cocycle determining the extension $G$ of $N$ by $H$, and let $n^{*}$ be a character of $N$. Then $n^{*} \circ f: H \times H \rightarrow T$ is also a symmetric cocycle, and therefore it determines an Abelian extension of $\boldsymbol{T}$ by $\boldsymbol{H}$. However, all such extensions split (one proof is given in [1, Proposition 18.4, Corollary 3]); this means that $n^{*} \circ f$ is a coboundary, and hence that $\exists$ a function $b_{n^{*}}: H \rightarrow T$, with

$$
\frac{b_{n^{*}}\left(h+h_{2}\right)}{b_{n^{*}}\left(h_{n}\right) b_{n^{*}}\left(h_{2}\right)}=\left\langle n^{*}, f\left(h_{1}, h_{2}\right)\right\rangle
$$

Define $g: N^{*} \times H \rightarrow T$ by

$$
g\left(n^{*}, h\right)=b_{n^{*}}(h) .
$$

The function $g$, of course is not uniquely determined by $f$. A straightforward computation shows that for fixed $n$ and $n_{2}, g\left(n_{1}^{*}+n_{2}^{*}, h\right) /\left(g\left(n_{1}^{*}, h\right) g\left(n_{2}^{*}, h\right)\right)$ is a 
character of $H$; call it $h^{*}(h)$. Define $f^{*}: N^{*} \times N^{*} \rightarrow H^{*}$ by $f^{*}\left(n_{1}^{*}, n_{2}^{*}\right)=h^{*}$. It is easily verified that $f^{*}$ is a symmetric cocycle.

THEOREM 3.1. The extension of $H^{*}$ determined by $f^{*}$ is $\cong G^{*}$.

Proof. We are given $G$ in the form of a compact open subgroup $N$, coset representatives $\boldsymbol{h}$ for each element $h$ of $H$, and a cocycle $f$. (See, e.g., [2, §15.2] for a discussion of how these items determine $G$.) Let our extension of $H^{*}$ by $N^{*}$ be $K$; in $K$, we have coset representatives $n^{*}$ for each element $n^{*}$ of $N^{*}$, and a cocycle $f^{*}$. We define a map $\psi: K \rightarrow G^{*}$ as follows:

On $H^{*}, \psi$ is the natural isomorphism onto $N^{\perp} \subseteq G^{*}$;

$\psi\left(\boldsymbol{n}^{*}\right)$ is the character of $G$ given by $\left\langle\psi\left(\boldsymbol{n}^{*}\right), n\right\rangle=\left\langle n^{*}, n\right\rangle,\left\langle\psi\left(\boldsymbol{n}^{*}\right), \boldsymbol{h}\right\rangle=g\left(n^{*}, h\right)$ ( $g$ is as in (3.1));

$\psi$ is extended on the $H^{*}$ cosets of $K$ by linearity.

Some simple computations show that $\psi$ is well-defined and a homomorphism; for instance, to show that $\psi\left(\boldsymbol{n}^{*}\right)$ is a character, note that

$$
\begin{aligned}
\left\langle\psi\left(n^{*}\right),\left(\boldsymbol{h}_{1}+\boldsymbol{h}_{2}\right)\right\rangle & =g\left(n, h_{1}+h_{2}\right)=g\left(n, h_{1}\right) g\left(n, h_{2}\right)\left\langle n, f\left(h_{1}, h_{2}\right)\right\rangle \\
& =\left\langle\psi\left(\boldsymbol{n}^{*}\right), \boldsymbol{h}_{1}+\boldsymbol{h}_{2}+f\left(h_{1}, h_{2}\right)\right\rangle .
\end{aligned}
$$

If $\psi(k)=0$, then $\psi(k)=0$ on $N$ and hence $k \in H^{*}$; also, $\psi(k)=0$ on $H$, and so $k=0$. Hence $\psi$ is 1-1. $\psi$ is open and continuous because $\psi \uparrow H^{*}$ is. Finally, if $x \in G$ is such that $\psi(K) \perp x$, then $x \in N$ (since $\psi\left(H^{*}\right)=N^{\perp}$ ), and hence $x=0$ (since otherwise $\left\langle\psi\left(\boldsymbol{n}^{*}\right), x\right\rangle \neq 1$ for some $\left.\boldsymbol{n}^{*}\right)$. Therefore $\psi(K)$ is dense in $G^{*}$; it follows that $\psi$ is an somorphism.

The two extensions are thus determined by the function $g$; conversely, it is easily checked that any function $g: N^{*} \times H^{*} \rightarrow T$ such that

$$
\begin{aligned}
& \text { (1) } \frac{g\left(n_{1}^{*}+n_{2}^{*}, h\right)}{g\left(n_{1}^{*}, h\right) g\left(n_{2}^{*}, h\right)} \text { is linear in } h ; \\
& \text { (2) } \frac{g\left(n^{*}, h_{1}+h_{2}\right)}{g\left(n^{*}, h_{1}\right) g\left(n^{*}, h_{2}\right)} \text { is linear in } n^{*} \text {; }
\end{aligned}
$$

determines a dual pair of extensions. We shall call such a function a coaxle (since it connects two cocycles). The next lemma shows when coaxles are equivalent.

LEMMA 3.2. Two coaxles $g_{1}, g_{2}$ determine the same extension $\Leftrightarrow \exists$ functions $b_{1}: N^{*} \rightarrow H^{*}$ and $b_{2}: H \rightarrow N$ such that $g_{1}\left(n^{*}, h\right)=g_{2}\left(n^{*}, h\right)\left\langle b_{1}(n), h\right\rangle\left\langle b_{2}(h), n^{*}\right\rangle$. In particular, the trivial coaxles are of the form $\left\langle b_{1}\left(n^{*}\right), h\right\rangle\left\langle b_{2}(h), n^{*}\right\rangle$. The coaxles mod trivial coaxles form a group isomorphic to the subgroup of $H^{2}(N, H)$ determining Abelian extensions.

Proof. The second half is obvious, given the first; the first half is easily checked by brute force.

The following lemmas are useful in analyzing coaxles:

Lemma 3.4. Suppose that $N^{*}=\sum_{\alpha \in \mathscr{A}} N_{\alpha}^{*}$ and $H=\sum_{\beta \in \mathscr{B}} H_{\beta}$ and that $g$ is a coaxle 
on $N^{*} \times H$; then there exist coaxles $g_{\alpha \beta}^{\prime}$ on each $N_{\alpha}^{*} \times H_{\beta}$ such that $g$ is equivalent to $g^{\prime}$, where $g^{\prime}\left(\sum n_{\alpha}^{*}, \sum h_{\beta}\right)=\prod_{\alpha, \beta} g_{\alpha \beta}^{\prime}\left(n_{\alpha}^{*}, h_{\beta}\right)$.

Proof. For each $n_{\alpha}^{*}$ of $N_{\alpha}^{*}$, define $b_{n_{\alpha}^{*}}$ as described before Theorem 3.1; then $\prod b_{n_{\alpha}^{*}}$ can serve as $b_{\sum n_{\dot{\alpha}}^{*}}$. Hence $g$ is equivalent to $g^{\prime \prime}$, where $g^{\prime \prime}\left(\sum n_{\alpha}, h\right)=\prod_{\alpha} g_{\alpha}\left(n_{\alpha}^{*} h\right)$. Repeat this argument with $H$ to get the result.

LeMmA 3.5. Let $0 \rightarrow N \rightarrow G \stackrel{\phi}{\rightarrow} H \rightarrow 0$ be an exact sequence, and let $g$ be $a$ coaxle on $N^{*} \times H$ which gives this extension. Suppose $M$ and $K$ are subgroups of $N^{*}$ and $H$ respectively. The restriction of $g$ to $M \times K$ gives the extension of $N / M^{\perp}$ by $K$ which is isomorphic to $\phi^{-1}(K) / M^{\perp}$.

Proof. If $M=N^{*}$, considering the cocycles makes the lemma clear; if $K=H$, the lemma follows from dualizing the first half (or, again, from arguments on cocycles). Now put these two statements together.

4. Symmetric coaxles. The case of greatest interest to us in this paper is that in which $N^{*}=H$.

THEOREM 4.1. The extension of $N$ by $N^{*}$ described by the coaxle $g$ is self-dual $\Leftrightarrow g$ is equivalent to a symmetric coaxle $g^{\prime}$ (i.e., $g^{\prime}$ satisfies $g^{\prime}\left(n_{1}^{*}, n_{2}^{*}\right)=g^{\prime}\left(n_{2}^{*}, n_{1}^{*}\right)$ ).

Proof. $\Leftarrow$ is trivial. For $\Rightarrow$, suppose that the associated cocycles $f$ and $f^{*} \operatorname{differ}$ by a coboundary $c$. Then we may write $f^{*}\left(n_{1}^{*}, n_{2}^{*}\right)=f\left(n_{1}^{*}, n_{2}^{*}\right)+c\left(n_{1}^{*}+n_{2}^{*}\right)-c\left(n_{1}^{*}\right)$ $-c\left(n_{2}^{*}\right)$. Let $g_{1}\left(n_{1}^{*}, n_{2}^{*}\right)=g\left(n_{1}^{*}, n_{2}^{*}\right)\left\langle c\left(n_{1}^{*}\right), n_{2}^{*}\right\rangle$; then $g$ and $g_{1}$, are equivalent, and the cocycles $f_{1}, f_{1}^{*}$ associated with $g_{1}$ are identical. Thus we may assume $f=f^{*}$.

Now let

$$
k\left(n_{1}^{*}, n_{2}^{*}\right)=g\left(n_{1}^{*}, n_{2}^{*}\right) / g\left(n_{2}^{*}, n_{1}^{*}\right) .
$$

It is easily checked that $k$ is antisymmetric (i.e., $k\left(n_{1}^{*}, n_{1}^{*}\right)=1$ and

$$
\left.k\left(n_{1}^{*}, n_{2}^{*}\right) k\left(n_{2}^{*}, n_{1}^{*}\right)=1\right)
$$

and bilinear. Suppose that we can write

$$
k\left(n_{1}^{*}, n_{2}^{*}\right)=l\left(n_{1}^{*}, n_{2}^{*}\right) / l\left(n_{2}^{*}, n_{1}^{*}\right),
$$

where $l$ is bilinear. Then $g\left(n_{1}^{*}, n_{2}^{*}\right) / l\left(n_{1}^{*}, n_{2}^{*}\right)$ is symmetric and equivalent to $g$, and the theorem is proved.

Thus we must prove that $l$ exists. By Proposition 6.1 of [5], $k$ can be written in the form

$$
k\left(n_{1}^{*}, n_{2}^{*}\right)=\frac{l_{0}\left(n_{1}^{*}, n_{2}^{*}\right)}{l_{0}\left(n_{2}^{*}, n_{1}^{*}\right)} k_{1}\left(n_{1}^{*}, n_{2}^{*}\right)
$$

where $k_{1}$ is both symmetric and antisymmetric and is constant on the cosets of $2 N^{*}$. [Note: there is a typographical error in the statement of the proposition cited above; its conclusion should read, "Then $A(G)=B^{(2)}(G) \cdot(A(G) \cap S(G))$."] It now suffices to show that

$$
\exists l_{1}: k_{1}\left(n_{1}, n_{2}\right)=l_{1}\left(n_{1}^{*}, n_{2}^{*}\right) / l_{1}\left(n_{2}^{*}, n_{2}^{*}\right)
$$


We may regard $k_{1}$ as defined on $N^{*} / 2 N^{*}$, and hence it suffices to prove the theorem in the case where $N^{*}$ is a direct sum of two-element groups. Let $N^{*}=\sum_{\alpha \in \mathscr{A}} N_{\alpha}^{*}$, where each $N_{\alpha}^{*}$ has two elements. Since $k_{1}$ is bilinear, we have $k_{1}\left(\sum n_{\alpha}, \sum m_{\beta}\right)$ $={ }_{\alpha, \beta} k_{1 \alpha \beta}\left(n_{\alpha}, m_{\beta}\right)$, where the sums and products are really finite. We know that $k_{1 \alpha \beta}\left(n_{\alpha}, m_{\beta}\right) k_{1 \beta \alpha}\left(m_{\beta}, n_{\alpha}\right)=1$; also $k_{1 \alpha \alpha} \equiv 1$, as a quick calculation shows. Well-order $\mathscr{A}$; set $l_{1}\left(\sum x_{\alpha}, \sum y_{\beta}\right)=\sum_{\alpha<\beta} k_{1 \alpha \beta}\left(x_{\alpha}, y_{\beta}\right)$. The theorem follows.

Lemma 4.2. Suppose $N=\prod_{\alpha \in \mathscr{A}} N_{\alpha}$, and $g$ is a symmetric coaxle on $N^{*} \times N^{*}$. Then $\exists$ coaxles $g_{\alpha \beta}$ on $N_{\alpha}^{*} \times N_{\beta}^{*}$, with $g_{\alpha \beta}=g_{\beta \alpha}$ and $g_{\alpha \alpha}$ symmetric, such that the coaxle $g^{\prime}\left(\sum n_{\alpha}^{*}, \sum m_{\beta}^{*}\right)={ }_{\alpha, \beta} g_{\alpha \beta}\left(n_{\alpha}^{*}, m_{\beta}^{*}\right)$ is equivalent to $g$.

Proof. By Lemma 3.4, $g$ is equivalent to a product of $g_{\alpha \beta}$ 's. Because $g\left\lceil N_{\alpha} \times N_{\alpha}\right.$ is equivalent to $g_{\alpha \alpha}$ (by Lemma 3.5) and $g$ is symmetric, we may take $g_{\alpha \alpha}$ to be symmetric. A similar argument shows that we may take $g_{\alpha \beta}\left(n_{\alpha}^{*}, m_{\beta}^{*}\right)=g_{\beta \alpha}\left(m_{\beta}^{*}, n_{\alpha}^{*}\right)$.

The problem of finding all self-adjoint extensions of a compact group by its dual now becomes the problem of finding all symmetric coaxles. In the next two sections, we give one classification of symmetric coaxles. In the remainder of this section, we give examples of symmetric coaxles; later we shall give one which describes a new self-dual group.

The problem of finding symmetric coaxles on $C\left(p^{m}\right) \times C\left(q^{n}\right)$ is not difficult; one can either use the defining relations (3.2) or the extensions themselves.

LeMma 4.3. If $p$ and $q$ are distinct primes, then every coaxle on $C\left(p^{m}\right) \times C\left(q^{n}\right)$ is trivial. Every coaxle on $C\left(p^{m}\right) \times C\left(p^{n}\right)$ is equivalent to one of the form $g(a, b)$ $=\exp (2 \pi i k a b), 0 \leqq k<p^{m+n-1}$. (Here, we are regarding $C\left(p^{m}\right)$ as a subgroup of $Q / Z$; see the end of $\S 1$.)

The proof involves straightforward computations and will be omitted.

LEMMA 4.4. Every coaxle of $\left(Z\left(p^{\infty}\right) \times Z\left(q^{\infty}\right)\right)$, with $p \neq q$, is trivial. If $p=q$, every coaxle is equivalent to one of the form $g(a, b)=\chi(k a b)$ for some $k \in Z_{p}$, where $\chi$ is the canonical character on $Z_{p}$ (see, for instance, [7, p. 66], for details).

Proof. Suppose first that $p \neq q$. Then we need to show that every extension of $\boldsymbol{Z}_{p}$ by $\boldsymbol{Z}\left(q^{\infty}\right)$ is trivial. Because multiplication by $q$ is an automorphism of $\boldsymbol{Z}_{p}$, multiplication by $q$ is a homeomorphism of the coset of $a \in Z\left(q^{\infty}\right)$ onto the coset of $q a$. It follows that if $a$ is of order $q^{n}$, then there is a unique element $a$ in the coset of $a$ with $q^{n} a=0$. The elements $\boldsymbol{a}$ form a group (because of the uniqueness) which splits the extension; this proves the theorem in that case.

For $p=q$, one can give a similar argument (looking at the extensions), or reason as follows: restricted to $C\left(p^{n}\right) \times C\left(p^{n}\right), f(a, b)$ can be written as

$$
\left\langle c_{1}(a), b\right\rangle\left\langle c_{2}(b), a\right\rangle \chi(k a b),
$$

where $c_{1}: Z\left(p^{n}\right) \rightarrow Z_{p}, c_{2}: Z\left(p^{n}\right) \rightarrow Z_{p}$, and $k \in Z_{p}$ is determined $\bmod p^{n-1}$. Now take limits.

The coaxle $g(a, b)=\chi\left(k_{1} p^{n} a b\right)$, with $k_{1}$ a unit of $Z_{p}$, determines an extension 
$\cong C\left(p^{n}\right) \oplus Q_{p}$, with the compact subgroup equal to the closure of the group generated by $\left(1 / p^{n}, k_{1}\right)$. For $n=\infty$ (or $\left.k=0\right)$, the extension splits.

5. A characterization. Our characterization of the self-dual groups under discussion follows from the following extension theorem:

THEOREM 5.1. Let $g$ be a symmetric coaxle on $G \times G$. Then there exists an Abelian group $G_{0}$ and a symmetric coaxle on $G_{0} \times G_{0}$ such that:

(1) $G \subseteq G_{0}$, and $g_{0}$ extends $g$.

(2) The torsion subgroup of $G_{0}$ is divisible (and hence a direct summand).

Proof. Let $G_{1}$ be the minimal divisible extension of $G$. Consider all pairs $\left(G_{\alpha}, g_{\alpha}\right)$ such that $G \subseteq G_{\alpha} \subseteq G_{1}$ and $g_{\alpha}$ is a symmetric coaxle on $G_{\alpha} \times G_{\alpha}$ which extends $g$. Then Zorn's lemma shows that there is a maximal pair $\left(G_{0}, g_{0}\right)$. We now have to show that the torsion subgroup of $G_{0}$ is divisible. If not, then $G_{0}$ contains a cyclic direct summand $H_{0} \cong C\left(p^{n}\right)$ for some prime $p$ (see, for instance, [4, Theorem 9]), and we may thus write $G_{0}=H_{0} \oplus H_{1}$. Let $H_{00}$ be a cyclic group of order $p^{n+1}$. We now show that $g_{0}$ can be extended to $G_{00}=H_{00} \oplus H_{1}$; from this, the theorem follows.

The extension of $g_{0}$ takes place in two steps: first we show that a coaxle equivalent to $g_{0}$ can be extended. For the first half, let $b$ be a trivial symmetric coaxle. Then there are functions $C_{1}, C_{2}: G_{0} \rightarrow G_{0}^{*}$ such that $b\left(x_{1}, y_{2}\right)=\left\langle C_{1}\left(x_{1}\right) y_{2}\right\rangle\left\langle C_{2}\left(x_{2}\right), x_{1}\right\rangle$. Furthermore, since $b$ is symmetric, the map $\left(x_{1}, y_{2}\right) \rightarrow\left\langle C_{1}\left(x_{1}\right)-C_{2}\left(x_{1}\right), y_{2}\right\rangle$ is symmetric and bilinear. Thus we may write

$$
b\left(x_{1}, y_{2}\right)=\left\langle C_{2}\left(x_{1}\right), y_{2}\right\rangle\left\langle C_{2}\left(y_{2}\right), x_{1}\right\rangle B\left(x_{1}, y_{2}\right),
$$

where $B$ is symmetric and bilinear. Extending $C_{2}$ to $G_{00}$ is trivial, since every character of $G_{0}$ extends to a character of $G_{00}$; the problem is extending $B$.

On $H_{0} \oplus H_{1}$, we may write

$$
B\left(h_{0}, h_{1}, h_{0}^{\prime}, h_{1}^{\prime}\right)=B_{0}\left(h_{0}, h_{0}^{\prime}\right) B_{1}\left(h_{0}, h_{1}^{\prime}\right) B_{1}\left(h_{1}, h_{0}^{\prime}\right) B_{2}\left(h_{1}, h_{1}\right) .
$$

$B_{2}$ does not need extending. For $B_{0}$, note that $B_{0}\left(h_{0}, h_{0}^{\prime}\right)=\exp \left(2 \pi i p^{n+k} h_{0} h_{0}^{\prime}\right)$ extend this to $H_{00}$ by using the same definition. (This extension is not necessarily bilinear, but it is a coaxle.) That leaves us with $B_{1}$; on it, we use a compactness argument. Let $k_{0}=p^{-n}$ and $k_{1}=p^{-n-1}$. Then $B_{1}$ is determined by the values of $A\left(h_{1}\right)=$ $B\left(k_{0}, h_{1}\right)$ for $h_{1} \in H_{1}$. We will define the extension $B_{01}$ of $B_{1}$ by $B_{01}\left(m k_{1}, h_{1}\right)$ $=\left[B_{01}\left(k_{1}, h_{1}\right)\right]^{m}$; thus $B_{01}\left(k_{1}, h_{1}\right)$ will be a $p$ th root of $A\left(h_{1}\right)$. For each $h_{1} \in H_{1}$, let $S\left(h_{1}\right)$ denote the set of $p$ th roots of $A\left(h_{1}\right)$, with the discrete topology, and let $S=\prod_{h_{1} \in H_{1}} S\left(h_{1}\right)$. For each finite set $T$ of elements of $H_{1}$, let $C(t)=\{a \in S:$ on the subgroup generated by $T$, the function given by $a$ is a coaxle $\}$. It is easily checked that $C(T)$ is closed and nonempty; the sets $C(T)$ satisfy the finite intersection property, since $C\left(T_{1} \cup T_{2}\right) \subseteq C\left(T_{1}\right) \cap C\left(T_{2}\right)$. By Tihonov's theorem, $\exists a \in \bigcap_{T} C(T)$. Let $B_{01}\left(k_{1}, h_{1}\right)=a\left(h_{1}\right)$.

So now we need only extend some coaxle equivalent to $g_{0}$. We may therefore 
assume that $g_{0}\left(h_{0}, h_{1} ; h_{0}^{\prime}, h_{1}^{\prime}\right)=f_{0}\left(h_{0}, h_{0}^{\prime}\right) f_{1}\left(h_{0}, h_{1}^{\prime}\right) f,\left(h_{1}, h_{0}^{\prime}\right) f_{2}\left(h_{1}, h_{1}^{\prime}\right)$. Also, by Lemma 4.3, we may assume that $f_{0}\left(h_{0}, h_{0}^{\prime}\right)=\exp \left(2 \pi i p^{r} h_{0} h_{0}^{\prime}\right)$ for some $r$. Extend $f_{0}$ to $H_{00}$ by using the same definition; $f_{2}$ does not need to be extended. As for $f_{1}$, it defines an extension $K_{0}$ of $H_{1}$ by $H_{0}$. To extend it, we need to show that there is an extension $K_{00}$ of $H_{1}$ by $H_{00}$ whose restriction to $H_{00}$ is $K_{0}$. This is easy. Let $k_{0}$ generate $H_{0}$, and suppose that $\boldsymbol{k}_{0}$ is a coset representative of $k_{0}$ in $K_{0}$. We extend $K_{0}$ by $C(p)$ to obtain $K_{00}$; the cocycle defining this relation is given by

$$
\begin{aligned}
(a / p, b / p) & =\boldsymbol{k}_{0} & & \text { if } a+b>\frac{1}{2} \\
& =-\boldsymbol{k}_{0} & & \text { if } a+b \leqq-\frac{1}{2} \\
& =0 & & \text { otherwise. }
\end{aligned}
$$

The theorem follows.

We can now apply Theorem 5.1 to get a classification theorem for some self-dual groups.

THEOREM 5.2. Let $G$ be a self-adjoint extension of a compact open subgroup $N$ by $N^{*}$. Then there exists a group $G_{0}$ with subgroups $G_{p}, N_{p}$ (one for each prime $p$ ), $N_{1}$ and $G^{\prime}$, such that:

(1) Each group $G_{p}$ is a self-adjoint extension of $N_{p}$ by $N_{p}^{*}$.

(2) $N_{p}$ is a direct product of copies of $Z_{p}$ 's (and hence $G_{p}$ is a p-group).

(3) $N_{1}$ is a divisible compact group.

(4) $G_{0}=N_{1} \times N_{0}^{*} \times \Pi^{\prime}\left(G_{p} ; N_{p}\right)$, a restricted direct product of the $G_{p}$ 's. Hence $G_{0}$ is a self-adjoint extension of $N_{1} \times \prod N_{p}=N_{0}$ by its dual.

(5) $N \subseteq N_{0} \subseteq G^{\prime}$, and, if $\phi: G_{0} \rightarrow G_{0}^{*}$ is the isomorphism from (4), then

$$
G^{\prime} \mid \phi^{-1}(G) \cong G
$$

Proof. We let $N_{0}^{*}$ be the group of Theorem 5.1. Because the torsion subgroup of $N_{0}^{*}$ is divisible, it is a direct sum of $Z\left(p^{\infty}\right)$ 's and is itself a direct summand. Let the other direct summand be $N_{1}^{*} ; N_{p}^{*}$ is the $p$-primary component of the torsion subgroup of $N_{0}^{*}$. Let $G_{0}$ be the extension of $N_{0}$ by $N_{0}^{*}$ obtained from the coaxle $g_{0}$, and let $G_{p}$ be the extension of $N_{p}^{*}$ by $N_{p}^{*}$ obtained by restricting $g_{0}$.

The coaxle $g_{0}$ may be written as a product of coaxles, each connecting some $N_{j}^{*}$ with a $N_{k}^{*}$. If $j=1$, then $N_{j}$ is divisible; as a result, every extension splits and the corresponding coaxle is trivial. If $j \neq k$, then Lemma 4.4 shows that the coaxle is trivial. Property (4) follows; (1)-(3) are clear. For property (5), use Lemma 3.5.

Theorem 5.2 provides a sort of characterization of the self-dual groups satisfying hypotheses (A) and (B). In the next section,we show why certain simpler characterizations would be unsatisfactory.

6. An example. In this section, we exhibit a self-dual group $G$ which cannot be constructed by the methods $1-5$ listed in $\S 1$. The example seems to indicate that a classification theorem along the lines of Theorem 5.2 is really necessary.

To describe the group $G$ we give a discrete group $N^{*}$ and a symmetric coaxle on $N^{*} \times N^{*}$. Pick a prime $p$, and let $N_{j}^{*}$ be the cyclic group on $p^{j}$ elements. Regard 
$N_{j}^{*}$ as a subgroup of $\boldsymbol{Q} / \boldsymbol{Z}$, and represent the elements of $N_{j}^{*}$ by their coset representatives in $\left(-\frac{1}{2}, \frac{1}{2}\right)$. Let $N^{*}$ be the subgroup of $\prod_{j=1}^{\infty} N_{j}^{*}$ consisting of all elements $\left(n_{1}^{*}, n_{1}^{*}, \ldots\right)$ such that $\sum_{j=1}^{\infty} n_{j}^{* 2}$ converges. Note that $N_{j}^{*} \subset N$ for all $j$ and that $\sum_{j=1}^{\infty} N_{j}^{*}=T^{*}$ is the torsion subgroup of $N^{*}$. Define the symmetric coaxle $g$ by $g\left(\left(n_{1}^{*}, \ldots\right),\left(m_{1}^{*}, \ldots\right)\right)=\exp \left(2 \pi i \sum_{j=1}^{\infty} n_{j}^{*} m_{j}^{*}\right)$. Then $f$ gives a self-adjoint extension $G$ of $N$ by $N^{*}$.

THEOREM 6.1. G cannot be written as $F \times G_{0}$, where $F$ is finite and $G_{0}=\prod_{\alpha}^{\prime}\left(G_{\alpha} ; H_{\alpha}\right)$ is a restricted direct product such that each pair $\left(G_{\alpha}, H_{\alpha}\right)$ is isomorphic to one of the following: $\left(C\left(p^{2 n}\right) ; C\left(p^{n}\right)\right),\left(Q_{p} ; Z_{p}\right)$, or $\left(K \oplus K^{*} ; C \oplus C^{\perp}\right)$, where $C$ is compact open in $K$.

Proof. 1. First of all, suppose that $F_{0}$ is a cyclic direct summand of order $p^{k}$. If $k$ is even, $F_{0}$ may be included in the restricted direct product; we now show that $k$ cannot be odd. Otherwise, let $M_{k}=\sum_{j=1}^{k} N_{j}^{*}$, and let $L_{k}$ be the subgroup of $N^{*}$ whose first $k$ coordinates are 0 . Then $N^{*}=M_{k} \oplus L_{k}$, and $f$ is the product of $f \mid L_{k} \times L_{k}=g_{k}$ (say) and $f \mid M_{k} \times M_{k}=h_{k}$. Therefore $G$ is a direct product of the groups $G_{k}, H_{k}$ determined by $g_{k}$ and $h_{k}$ respectively. Furthermore, Lemma 3.2 says that one of these groups contains a cyclic direct summand of order $p^{k}$. It is easily checked that $H_{k}$ is the direct sum of cyclic groups of order $p^{2}, \ldots, p^{2 k}$; since $k$ is odd, $G_{k}$ must contain a cyclic direct summand of order $p^{k}$, which we call $F_{1}$. If $F_{1} \cap L_{k}^{*}=\{0\}$, then $F$ may be regarded as in $L_{k}$; this is clearly impossible, since $L_{k}$ contains no pure subgroup of order $p^{k}$. Otherwise, let $\chi$ be a character on $G_{k}$ which is $\exp \left(2 \pi i / p^{k}\right)$ on some generator of $F$, and is trivial on the other summand. Then $\chi \mid L_{k}^{*}$ is an element of $L_{k}$ which is of order dividing $p^{k}$ and which is not divisible by $p$; this, too is impossible. Hence $k$ is even. Therefore we may assume $F=\{0\}$.

2. The preceding argument shows that $G$ has no cyclic direct summands of order $p^{2 n+1}$; it is clear from similar reasoning that $G$ cannot be written as $C_{0} \oplus C_{1} \oplus G^{\prime}$, where $C_{0}$ and $C_{1}$ are both cyclic of order $p^{2 n}$.

3. Now suppose that the theorem is false and $G \cong \prod_{\alpha}^{\prime}\left(G_{\alpha} ; H_{\alpha}\right)$. For each $n$, $G$ contains a cyclic direct summand of order $p^{2 n}$. Hence, by Lemma 2.2, some $G_{\alpha}$ does. Clearly $G_{\alpha}$ cannot be $\cong Q_{p}$; if $G_{\alpha} \cong K \oplus K^{*}$, then $G_{\alpha}$ must contain two direct summands of order $p^{2 n}$ and this is impossible. So some $G_{\alpha} \cong C\left(p^{2 n}\right)$.

4. Suppose that for some $\alpha, G_{\alpha}$ is not a cyclic $p$-group; we show that $H_{\alpha}^{*}$ contains no torsion element of finite height. If it does, then [4, Theorem 9] $H_{\alpha}^{*}$ contains a finite cyclic direct summand, and hence $H_{\alpha}=C \oplus C^{\perp}$ does. Call this summand $F_{\alpha}$. If $F_{\alpha} \cap N=\{0\}$, then $F_{\alpha}$ (as in part 1 ) is a cyclic $p$-group and a direct summand of $F$; we have already accounted for all such subgroups in parts 2 and 3 , and thus this case is impossible. If $F_{\alpha} \cap N \neq\{0\}$, the discussion of part 1 shows that $F_{\alpha} \cap N$ is contained in a cyclic direct summand of order $p^{2 n}$. This, too, is impossible by the earlier discussion. It follows that the torsion subgroup of $H_{\alpha}^{*}$ is divisible and hence a direct summand. 
5. Now let $H=\prod_{\alpha} H_{\alpha}$. Then the torsion subgroup of $H^{*}$ is a direct summand of $H^{*}$ (since this holds for each $H_{\alpha}^{*}$ ). Since $N$ and $H$ are compact open, $N \cap H$ has finite index in $N$ and in $H$; thus Lemma 2.2 shows that the torsion subgroup $T^{*}$ of $N^{*}$ is a direct summand.

Now comes the contradiction. It is clear that no element of $N^{*}$ (except 0 ) has infinite height. But the image of $\left(1 / p, 1 / p, 1 / p^{2}, 1 / p^{2}, 1 / p^{3}, \ldots\right)$ in $N^{*} / T^{*}$ does have infinite height. Hence $N^{*} / T^{*}$ is not isomorphic to any subgroup of $N^{*}$, and so it is not a direct summand. The theorem follows.

7. Concluding remarks. As remarked in $\$ 1$, it would be nice to see if either hypothesis (A) or (B) could be dispensed with. The other obvious point at which this paper is incomplete is the characterization of the groups $G_{p}$ in Theorem 5.2. It seems plausible to conjecture that $G_{p}$ can be written as a restricted direct product of groups of the form $Z_{p} \times Z\left(p^{\infty}\right)$ or $\boldsymbol{Q}_{p} \times C\left(p^{n}\right)$ (where in the latter case, the compact open subgroup is the closure of the group generated by $\left.\left(1,1 / p^{n}\right)\right)$. In cases where $N_{p}$ is a finite product of copies of $Z_{p}$, it is not hard to prove this by extending Lemma 3.4. But in general, it seems more difficult. Perhaps the following will make the problem clearer. Suppose that we have a coaxle on $\boldsymbol{Z}\left(p^{\infty}\right)^{\wedge} \times \boldsymbol{Z}\left(p^{\infty}\right)^{\wedge}$; writing it as a product of coaxles on the $Z\left(p^{\infty}\right)$ factors and applying Lemma 4.4, we see that the coaxle can be described by a symmetric $\boldsymbol{W} \times \boldsymbol{W}$ matrix over $Z_{p}$. We want to know, essentially, if this can be diagonalized. In this case, the answer seems unclear.

In a recent paper [8], all self-dual torsion-free metric LCA groups were determined. They all satisfy hypotheses (A) and (B), and can be constructed from the rules listed in $§ 1$.

\section{BIBLIOGRAPHY}

1. L. Calabi, Sur les extensions des groupes topologiques, Ann. Mat. Pura. Appl. (4) 32 (1951), 295-370. MR 14, 245.

2. M. Hall, The theory of groups, Macmillan, New York, 1959. MR 21 \#1996.

3. E. Hewitt and K. A. Ross, Abstract harmonic analysis. Vol. I: Structure of topological groups. Integration theory, group representations, Die Grundlehren der math. Wissenschaften, Bd. 115, Academic Press, New York and Springer-Verlag, Berlin, 1963. MR 28 \#158.

4. I. Kaplansky, Infinite abelian groups, Univ. of Michigan Press, Ann Arbor, 1954. MR 16, 444.

5. A. Kleppner, Multipliers on abelian groups, Math. Ann 158 (1965), 11-34. MR 30 \#4856.

6. E. H. Spanier, Algebraic topology, McGraw-Hill, New York, 1966. MR 35 \#1007.

7. A. Weil, Basic number theory, Springer-Verlag, New York, 1967. MR 383244.

8. M. Rajagopalan and T. Soundarajan, Structure of self-dual torsion-free metric LCA groups, Fund. Math. 65 (1969), 309-316.

MassachusetTs Institute of TeChNOLOGY, Cambridge, Massachusetts 02139 